\title{
Selective Sorption and Desorption of Organic Solvent for $\delta$-Syndiotactic Polystyrene
}

\author{
By Takahiko NAKAOKI, ${ }^{*}$ Naoko GoTO, and Kohji SAITO
}

The desorption and sorption kinetics of organic solvents in the cavity of $\delta$ - and empty $\delta$-forms of syndiotactic polystyrene (sPS) were investigated as a function of molar volume and shape of the solvent molecules. The desorption process was performed for films cast from chloroform, benzene, chlorobenzene, toluene, and $p$-xylene in supercritical $\mathrm{CO}_{2}$ at $40{ }^{\circ} \mathrm{C}$ and $10 \mathrm{MPa}$. The nanoporous structure of $\delta$-sPS after extraction of solvent molecules was dependent on the solvent used. Large size solvent molecules were slowly excluded from $\delta$-sPS. The relation between the cavity volume of $\delta$-sPS and the dimensions of the solvent molecule suggests that desorption is slow for solvent molecules that are tightly packed in the cavities. The sorption kinetics of alcohols was investigated as a function of chain length using the nanoporous structure of empty $\delta$-sPS. When empty $\delta$-sPS was soaked in a linear alcohols with carbon numbers from 1 to 10 , the alcohols with carbon number less than 6 could be incorporated in the cavity of empty $\delta$-sPS. The diffusion coefficient of alcohols into the cavity was estimated using the Fickian equation. The diffusion became slower for longer chain length alcohols. In order to compare the sorption of different molecular shaped alcohols, structural isomers of 4 and 5 carbon number alcohols were investigated. These isomers were classified into two categories; alcohols with hydroxyl side groups and those with methyl side groups. Alcohols with hydroxyl side groups could be incorporated into empty $\delta$-sPS; however, the methyl side group prevented the insertion of alcohol molecules into the cavity of empty $\delta$-sPS.

KEY WORDS: Syndiotactic Polystyrene / $\delta$-Form / Supercritical Carbon Dioxide / Desorption and Sorption /

Syndiotactic polystyrene (sPS) has been given much attention in research, because of its polymorphic behavior. In particular, the complexation structure between sPS and solvent molecules has a unique character. The molecular conformation of the complex form is characterized by $\mathrm{at}_{2} \mathrm{~g}_{2}$ sequence, which is referred to as an eight-shaped helix. The solvent molecule plays an important role in the construction of this helical form. It has been reported that this complex form, known as $\delta$-sPS, can be prepared by casting from an sPS solution, or treating glassy sPS in a solvent vapour. ${ }^{1-8}$ In 1995, Corradini et al. found that an emptied clathrate form of sPS, which is abbreviated as empty $\delta$-form, can be obtained by extraction of solvent from the clathrate $\delta$-form in boiling acetone. ${ }^{9}$ Later, supercritical $\mathrm{CO}_{2}\left(\mathrm{scCO}_{2}\right)$ was used to extract the solvent. ${ }^{10,11}$ As for the crystalline structure, both $\delta$ - and empty $\delta$-forms take a monoclinic unit cell, but the b-axis of empty $\delta$-sPS is reduced as a consequence of guest molecule removal. ${ }^{9}$ Empty $\delta$-sPS takes a nanoporous structure after the extraction of solvent molecules. When empty $\delta$-sPS is soaked in liquid or exposed to the vapor of organic solvents such as halogenated compounds, then the solvent molecules can be incorporated into the cavities of empty $\delta$-sPS. It has been suggested that the nanoporous structure can be applied for practical uses such as separation and sensing. ${ }^{12,13}$

Guerra et al. reported the desorption and sorption kinetics of solvent molecules between the $\delta$ - and empty $\delta$-forms. ${ }^{14-17}$ The sorption rate was improved for a porous aerogel with mesh structure, which was prepared by treating the sPS physical gel in $\mathrm{scCO}_{2}$. The relationship between the size of the solvent molecules and the cavity volume of empty $\delta$-sPS was reported by Tsujita et al. ${ }^{18-21}$ They revealed that the cavity volume of $\delta$-sPS is reduced after extraction of the solvent molecules, and the cavity can include solvents with smaller spatial volumes than the cavity volume. Kaneko et al. investigated the guest exchange process for chloroform and $n$-alkanes in $\delta$-sPS, and it was shown that chloroform molecules in $\delta$-sPS can be replaced by $n$-alkanes. ${ }^{22-24}$

In this paper, the desorption kinetics of $\delta$-sPS with various molar volumes of solvent were investigated in $\mathrm{scCO}_{2}$. In addition, the sorption kinetics were measured for alcohols with various chain length and molecular shape.

\section{EXPERIMENTAL}

sPS was supplied from Idemitsu Co. Ltd. The weight average molecular weight $\left(M_{\mathrm{w}}\right)$ and polydispersity $\left(M_{\mathrm{w}} / M_{\mathrm{n}}\right)$ were $2.4 \times 10^{5}$ and 2.2, respectively. Empty $\delta$-sPS was prepared by casting from chloroform, benzene, chlorobenzene, toluene, and $p$-xylene solutions. Treatment in $\mathrm{scCO}_{2}$ was performed using supercritical fluid reaction apparatus (Jasco SCF-Get) under mild conditions of $40^{\circ} \mathrm{C}$ and $10 \mathrm{MPa}$. Fourier transform infrared (FT-IR) spectroscopy was measured using a Jasco FT-IR 660 Plus spectrometer with a DTGS detector. FTIR measurements were carried out at room temperature after treating in $\mathrm{scCO}_{2}$, and 32 transients were collected for each spectrum. In order to compare films with different thickness, 
the $1028 \mathrm{~cm}^{-1}$ band was used as an internal standard band; this band is assignable to in-plane mode $\mathrm{C}-\mathrm{H}$ bending for the phenyl group, which is a local mode irrespective of the main chain conformation. The calibration curve between the film thichness and the absorbance for $1028 \mathrm{~cm}^{-1}$ band was reported in our previous paper. ${ }^{25}$ The absorbance of the objective vibrational mode was normalized to a thickness of $50 \mu \mathrm{m}$. Wide-angle X-ray diffraction patterns were obtained on an automatic Rigaku RINT2500 diffractometer with Ni-filtered $\mathrm{Cu} \mathrm{K} \alpha$ radiation, using $\mathrm{NaF}$ as an internal standard in order to obtain accurate cell constants.

\section{RESULTS AND DISCUSSION}

\section{Desorption Kinetics of Solvent from $\delta$-sPS in $\mathrm{scCO}_{2}$}

The desorption kinetics of solvent in $\delta$-sPS were investigated in $\mathrm{scCO}_{2}$. Five different solvents were used for preparation of nanoporous empty $\delta$-sPS. In order to estimate the amount of residual solvent in the film, infrared spectra were measured before and after treatment in $\mathrm{scCO}_{2}$. The frequency modes of solvent isolated from sPS were monitored at 1219 , 678, 468, 728, and $798 \mathrm{~cm}^{-1}$ for chloroform, benzene, chlorobenzene, toluene, and $p$-xylene, respectively. The 1219 $\mathrm{cm}^{-1}$ band for chloroform is assignable to the $\mathrm{C}-\mathrm{H}$ deformation mode, and the absorption bands for aromatic compounds are assumed to the ring $\mathrm{C}-\mathrm{H}$ out-of-plane mode. In Figure 1, the absorbance of these characteristic bands, normalized by that of the as-cast film, was plotted against the time treated in $\mathrm{scCO}_{2}$. The band intensity decreased with increasing time, depending on the solvent used. In order to compare the desorption rate, the diffusion coefficient was estimated by the Fickian equation, as previously shown. ${ }^{14-16,26}$ The Fickian equation is expressed as

$$
\frac{\mathrm{A}(\mathrm{t})}{\mathrm{A}_{0}}=\frac{4}{\mathrm{~d}} \sqrt{\frac{\mathrm{Dt}}{\pi}},
$$

where $\mathrm{A}_{0}$ is the absorbance before treatment in $\mathrm{scCO}_{2}$ and $\mathrm{A}(\mathrm{t})$ is the absorbance depending on time $t$ after treatment in $\mathrm{scCO}_{2}$.

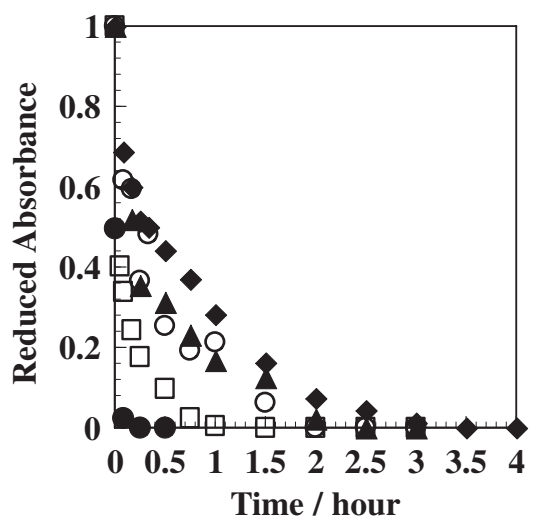

Figure 1. Time dependence of the characteristic absorption bands for chloroform $(\boldsymbol{O})$, benzene $(\square)$, chlorobenzene $(\boldsymbol{\Delta})$, toluene $(\bigcirc)$, and $p$-xylene $(\bullet)$ during the desorption process in $\mathrm{scCO}_{2}$. Each absorbance was normalized by that of the as-cast film.

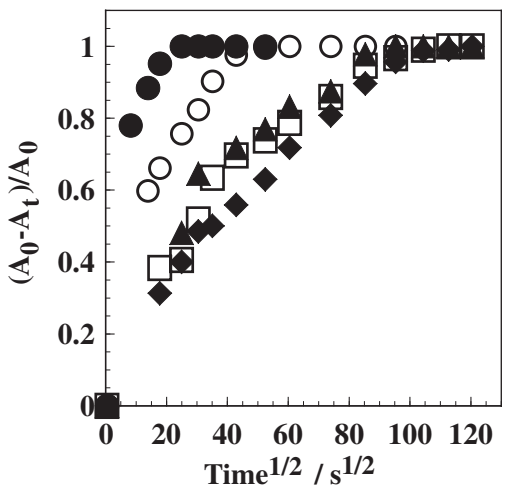

Figure 2. Absorbance vs. the square root of time for different molar volume solvents during the desorption process. 0 : chloroform, $\square$ : benzene, $\boldsymbol{\Delta}$ : chlorobenzene, $\bigcirc$ : toluene, and $\diamond$ : $p$-xylene.

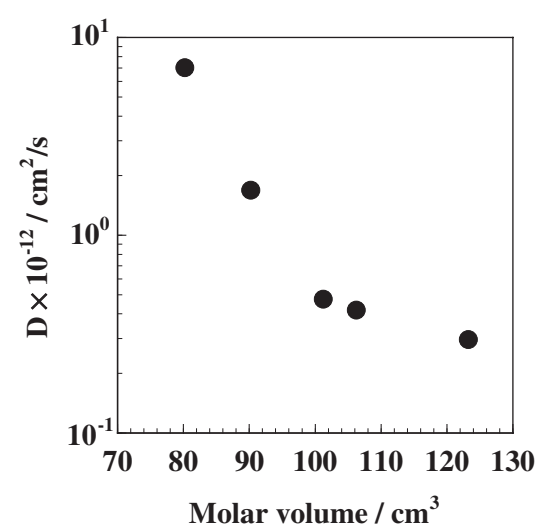

Figure 3. Diffusion coefficient for different molar volume solvents during the desorption process.

$\mathrm{D}$ and d denote the diffusion coefficient and the film thickness, respectively. For the desorption process, the absorbance $\mathrm{A}(\mathrm{t})$ in eq (1) was replaced by $\mathrm{A}_{0}-\mathrm{A}(\mathrm{t})$, and plotted against $\mathrm{t}^{1 / 2}$, as shown in Figure 2. The initial slope provides the apparent diffusion coefficient, which contains crystalline and noncrystalline phases. In this paper, the apparent diffusion coefficient averaged by these phases will be used to discuss the desorption and sorption processes. The cavity volume of empty $\delta$-sPS has a close relationship with the volume occupied by a solvent molecule. In Figure 3 the diffusion coefficient is plotted as a function of molar volume. Solvents with small molar volume, such as chloroform, had diffusion coefficient as large as $7.2 \times 10^{-12} \mathrm{~cm}^{2} / \mathrm{s}$, which decreased with the increase in molar volume. Guerra et al. estimated the diffusion coefficient of chloroform for desorption and sorption processes under a condition of reduced pressure. ${ }^{16}$ The desorption process at the initial stage, which corresponds to that of the present analysis, provided diffusion coefficients between $1.5 \times 10^{-12}$ and $6.2 \times$ $10^{-12}$, depending on the pressure. These values are comparable with those estimated by the measurements in $\mathrm{scCO}_{2}$.

The diffusion coefficients of large solvent molecules such as aromatic compounds were small. It was considered that the large solvent molecules are tightly packed in the crystalline 


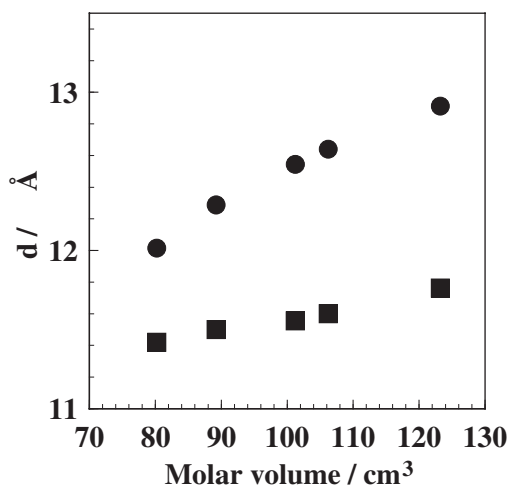

Figure 4. b-Axis of SPS films cast from chloroform, benzene, chlorobenzene, toluene, and $p$-xylene, before $(0)$ and after $(\square)$ treatment in $\mathrm{scCO}_{2}$.

unit of $\delta$-sPS. Therefore, X-ray diffraction patterns were measured to elucidate the cavity volume. As reported previously, the b-axis length can be used as an index of cavity volume, because the crystal structures of $\delta$ - and empty $\delta$-sPS have almost the same dimensions for the a and $\mathrm{c}$ axes, but only the $b$ axis is reduced after extraction of solvent molecules. ${ }^{9}$ Fortunately, the (010) reflection for both $\delta$ - and empty $\delta$-forms is strong and isolated from other diffraction peaks, allowing accurate estimation of the b-axis length.

Figure 4 shows the b-axis length as a function of molar volume of solvent used for preparation of the cast films. As previously reported, the b-axis depends on the size of solvent, and becomes longer for large solvent molecules. ${ }^{9,17,20}$ After extraction of the solvent molecule, the b-axis was reduced, but was still dependent on the molar volume. Guerra et al. reported the relationship between the b-axis length and the cavity volume ${ }^{27}$ the cavity volumes evaluated from the b-axis (Figure 5 in ref 27) were 116 and $153 \mathrm{~A}^{3}$ for chloroform and $p$-xylene, respectively. The cavity volume for $p$-xylene was approximately 1.3 times larger than that of chloroform, whereas the molar volume of $p$-xylene was approximately 1.5 times larger than that of chloroform. This indicates that the larger solvent molecule occupied a larger content in the cavity volume, which results in a smaller diffusion coefficient for large solvent molecules.

\section{Sorption Kinetics of Normal Alcohols as a Function of Chain Length}

It has been reported that some organic solvents can be incorporated in the cavity of empty $\delta$-sPS. In this section, the empty $\delta$-sPS obtained from casting of a chloroform solution was used, and the sorption of normal alcohols was investigated as a function of chain length. It is worth noting here that alcohol is a poor solvent for sPS, and therefore, alcohol molecules do not induce either crystallization or expansion of the cavity volume.

Figure 5 shows the infrared spectra of $\delta$-sPS soaked in linear alcohols, from methanol to $n$-decanol, for $24 \mathrm{~h}$. The $\mathrm{OH}$ stretching mode due to alcohol at around $3650 \mathrm{~cm}^{-1}$ was used to confirm the sorption of alcohol in the cavity of empty $\delta$-sPS.

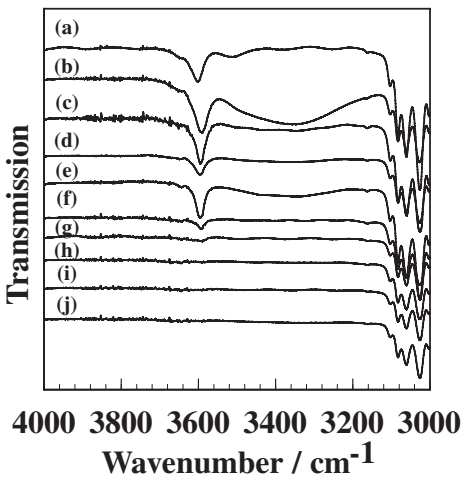

Figure 5. Infrared spectra of empty $\delta$-sPS after soaking in various chain length alcohols for $24 \mathrm{~h}$. (a) Methanol, (b) ethanol, (c) propanol, (d) $n$-butanol, (e) $n$-pentanol, (f) $n$-hexanol, (g) $n$-heptanol, (h) $n$ octanol, (i) $n$-nonanol, and (j) $n$-decanol.

In general, the frequency of the $\mathrm{OH}$ stretching mode varies depending on the state of hydrogen bonding. A liquid alcohol provides two peaks around 3360 and $3650 \mathrm{~cm}^{-1}$; the former peak is related to strong hydrogen bonding, whereas the latter corresponds to the isolated mode without hydrogen bonding. The $\mathrm{OH}$ stretching mode shown in Figure 5 was observed around $3650 \mathrm{~cm}^{-1}$, which can be assumed to be the vibrational mode without hydrogen bonding. When the alcohol molecule is clathrated in the cavity of empty $\delta$-sPS, the alcohol molecule is isolated from others. Therefore the appearance of the $\mathrm{OH}$ mode around $3650 \mathrm{~cm}^{-1}$ provides direct evidence that the alcohol molecule is incorporated in the cavity of empty $\delta$-sPS.

Alcohol molecules with less than 6 carbons provide an $\mathrm{OH}$ stretching mode in the infrared spectrum, indicating the sorption of shorter chain length alcohols. The sorption kinetics for methanol to $n$-pentanol were investigated by monitoring the time dependence of infrared spectroscopy. In Figure 6, the absorbance of the $\mathrm{OH}$ stretching mode was plotted against the time soaked in the liquid alcohol. The maximum absorbances were different and dependent on the chain length, as listed in Table I. According to the Beer-Lambert law, the absorbance of the $\mathrm{OH}$ stretching mode is proportional to the concentration of alcohol incorporated in $\delta$-sPS. Longer chain length alcohols result in larger absorbance of the $\mathrm{OH}$ stretching mode. This indicates that methanol is less incorporated in the empty $\delta$-sPS, compared with the larger chain length alcohols. This might be explained by hydrophilic interaction between sPS and the hydroxyl group of the alcohol; however, interpretation of this result is yet to be clarified, and further experiments will be required.

The diffusion coefficient was estimated using the Fickian equation shown in eq (1). The absorbance, normalized by the maximum, is plotted as a function of $\mathrm{t}^{1 / 2}$ in Figure 7 , where the initial slope provides the diffusion coefficient. The sorption became slower with the increasing chain length of the alcohols. The diffusion coefficient is plotted against the alcohol carbon number in Figure 8. Methanol provided the highest diffusion coefficient, because the size of methanol is smaller than the cavity volume of empty $\delta$-sPS. Another possible explanation is 


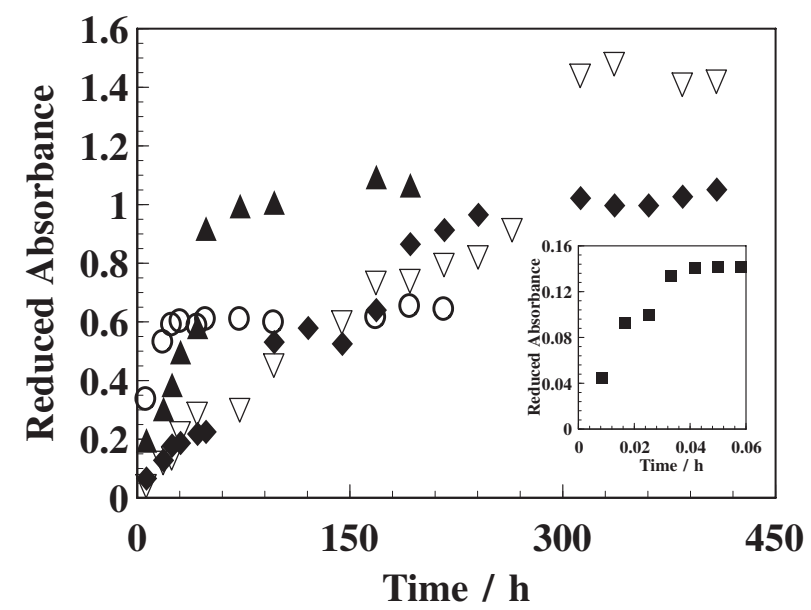

Figure 6. $\mathrm{OH}$ stretching mode absorptions for various chain length alcohols as a function of soaking time. The result for methanol is shown separately as an inset, due to the small absorbance compared with the other alcohols. 0 : methanol, $\bigcirc$ : ethanol, $\mathbf{\Delta}$ : propanol, $\nabla: n$-butanol, and $\diamond: n$-pentanol.

Table I. Maximum absorbance of the $\mathrm{OH}$ stretching mode after soaking the empty $\delta$-sPS film in liquid alcohols

\begin{tabular}{ccc}
\hline Solvent & Molar volume $/ \mathrm{cm}^{3}$ & Maximum absorbance \\
\hline Methanol & 80 & 0.14 \\
Ethanol & 89 & 0.62 \\
Propanol & 101 & 1.04 \\
Butanol & 106 & 1.02 \\
Pentanol & 123 & 1.44 \\
\hline
\end{tabular}

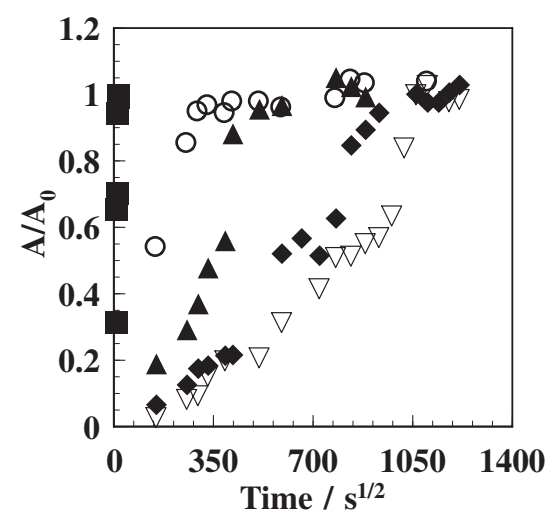

Figure 7. Normalized $\mathrm{OH}$ stretching mode absorptions for various chain length alcohols as a function of $\mathrm{t}^{1 / 2}$. : methanol, $\bigcirc$ : ethanol, $\mathbf{\Delta}$ : propanol, $\nabla: n$-butanol, and $\diamond$ : $n$-pentanol.

the contribution from molecular conformation. Longer chain length alcohols can assume a gauche conformation, although the probability is not so high, which could also cause slow diffusion.

\section{Sorption of Branched Alcohols}

In the previous section, normal alcohols were used to investigate the sorption kinetics as a function of carbon number. Normal alcohols are regarded as having a cylindrical shape, with a diameter that is the same, irrespective of the

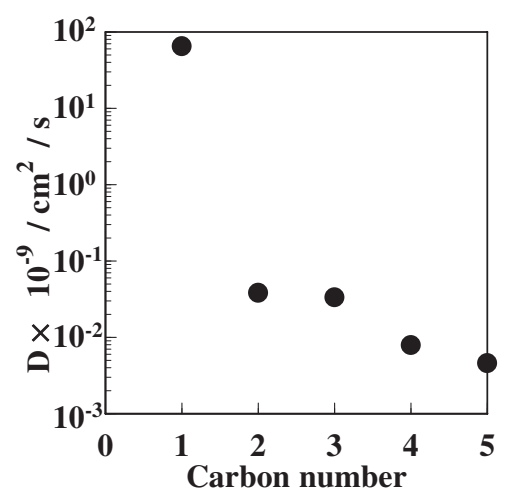

Figure 8. Diffusion coefficient for different chain length alcohols during the sorption process.

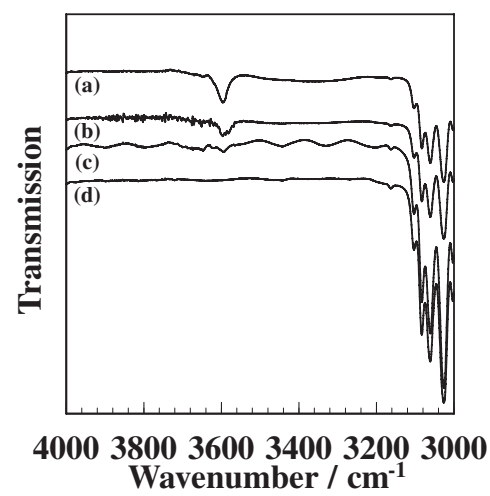

Figure 9. Infrared spectra of empty $\delta$-sPS after soaking in 4 carbon branched alcohols. (a) 1-Butanol, (b) 2-butanol, (c) 2-methyl-2propanol, and (d) 2,2-dimethyl-ethanol.

chain length. Therefore, normal alcohols can be incorporated in the cavity of empty $\delta$-sPS, irrespective of their chain length, although the diffusion coefficient is dependent on the chain length. In this section, the sorption of branched alcohols with the same carbon number was investigated.

Figure 9 shows the infrared spectra of sPS film after soaking in various 4 carbon alcohols for $24 \mathrm{~h}$. The $\mathrm{OH}$ stretching mode around $3650 \mathrm{~cm}^{-1}$ was observed for 1- and 2-butanols, but not for 2-methylpropanol and 1,1-dimethylethanol (tert-butanol). This indicates that the two former isomers were incorporated in the cavity of empty $\delta$-sPS, but the latter were not. Even after the empty $\delta$-sPS film was soaked in the latter alcohols for four days, there was no trace of the $\mathrm{OH}$ stretching mode. These results indicate that steric effects of the methyl side group prevents the alcohol molecules from being incorporated in the cavity of empty $\delta$-sPS.

Incorporation of branched alcohols with 5 carbons was also investigated by FT-IR spectroscopy. Figure 10 shows the infrared spectra of sPS films after soaking in pentanol isomers. The $\mathrm{OH}$ stretching mode was observed for 1-, 2-, and 3pentanols, which have linear methylene chains with hydroxyl side groups. However, no $\mathrm{OH}$ stretching mode was observed for 2-methyl-1-butanol and 2-methyl-2-butanol. These alcohols have a methyl side group. This result is the same as that for the 4 carbon alcohols. Therefore, it is concluded that alcohols with 


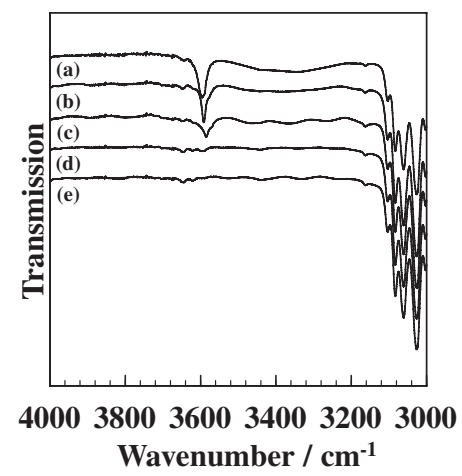

Figure 10. Infrared spectra of empty $\delta$-sPS after soaking in 5 carbon branched alcohols. (a) 1-Pentanol, (b) 2-pentanol, (c) 3-pentanol, (d) 2-methyl-1-butanol, and (e) 2-methyl-2-butanol.

hydroxyl side groups are small enough to be incorporated in the cavity of empty $\delta$-sPS, but alcohols with methyl side groups are too large to be incorporated in the cavity due to steric effets.

\section{CONCLUSIONS}

The desorption and sorption kinetics of organic solvents in $\delta$ - and empty $\delta$-sPS were investigated by infrared spectroscopy. The clathrate form of sPS was prepared by casting homogeneous solutions dissolved in chloroform, benzene, chlorobenzene, toluene, and $p$-xylene. The $\mathrm{b}$ axis of the $\delta$-form was enlarged with increasing molar volume of the solvent molecules. The desorption kinetics of these solvent molecules in $\delta$ sPS were investigated in $\mathrm{scCO}_{2}$. The residual solvent in $\delta$-sPS was monitored by characteristic absorption bands of these organic solvents in the infrared spectra. The diffusion coefficient of solvent molecules extracted from $\delta$-sPS was estimated by the Fickian equation. Diffusion became slow with increasing molar volume of the solvents, which might be explained by the size of the occupied solvent in the cavity of empty $\delta$-sPS; the large size solvents would be tightly packed in the cavity.

The sorption kinetics were investigated by soaking the empty $\delta$-sPS cast from chloroform solution in various types of different chain length normal alcohols for $24 \mathrm{~h}$. Incorporation of alcohol in the cavity of empty $\delta$-sPS was monitored by the $\mathrm{OH}$ stretching mode due to alcohol, which is assignable to the isolated alcohol without hydrogen bonding. The $\mathrm{OH}$ stretching mode was observed from methanol to $n$-hexanol, indicating that these alcohols were incorporated in the cavity of empty $\delta$-sPS. Longer chain length alcohols were comparatively slow to enter into the cavity of empty $\delta$-sPS, which can be interpreted by the effects of molecular size and conformation. In order to confirm the sorption for different shaped alcohols, branched alcohols with 4 and 5 carbons were used. For alcohols with the $\mathrm{OH}$ group attached as a side group, incorporation in the cavity of empty $\delta$-sPS occurred. However, those alcohols with methyl side groups could not be incorporated in the empty $\delta$-sPS, due to the bulkiness of the methyl side group.
Acknowledgment. This work was partially supported by a grant from the High-Tech Research Center Program for private universities by the Japan Ministry of Education, Culture, Sports, Science and Technology.

Received: September 5, 2008

Accepted: November 24, 2008

Published: January 21, 2009

\section{REFERENCES}

1. M. Kobayashi, T. Nakaoki, and N. Ishihara, Macromolecules, 22, 4377 (1989).

2. M. Kobayashi, T. Nakaoki, and N. Ishihara, Macromolecules, 23, 78 (1990).

3. T. Nakaoki and M. Kobayashi, J. Mol. Struct., 242, 315 (1991).

4. Y. Chatani, Y. Shimane, T. Inagaki, T. Ijitsu, T. Yukinari, and H. Shikuma, Polymer, 34, 1620 (1993).

5. Y. Chatani, T. Inagaki, Y. Shimane, and H. Shikuma, Polymer, 34, 4841 (1993).

6. A. Immirzi, F. De Candia, P. Iannelli, V. Vittoria, and A. Zambelli, Makromol. Chem. Rapid Commun., 9, 761 (1988).

7. G. Guerra, P. Musto, F. E. Karasz, and W. J. MacKnight, Makromol. Chem., 191, 2111 (1990).

8. V. Vittoria, Polym. Commun., 31, 263 (1990).

9. C. De Rosa, G. Guerra, V. Petraccone, and B. Pirozzi, Macromolecules, 30, 4147 (1997).

10. Y. P. Handa, Z. Zhang, and B. Wong, Macromolecules, 30, 8499 (1997).

11. E. Reverchon, G. Guerra, and V. J. Venditto, J. Appl. Polym. Sci., 74, 2077 (1999).

12. C. Daniel, D. Alfano, V. Venditto, S. Cardea, E. Reverchon, D. Larobina, G. Mensitieri, and G. Guerra, Adv. Mater., 17, 1515 (2005).

13. P. Stegmaier, A. De Girolamo, V. Venditto, and G. Guerra, Adv. Mater., 17, 1166 (2005).

14. C. Manfredi, M. A. Del Nobile, G. Mensitiere, G. Guerra, and M. J. Rapacciuolo, J. Polym. Sci., Part B: Polym. Phys., 35, 133 (1997).

15. L. Guadagno, P. Baldi, V. Vittoria, and G. Guerra, Macromol. Chem. Phys., 199, 2671 (1998).

16. P. Musto, G. Mensitieri, S. Cotugno, G. Guerra, and V. Venditto, Macromolecules, 35, 2296 (2002).

17. A. R. Albunia, A. Grassi, G. Milano, P. Rizzo, V. Venditto, P. Musto, and G. Guerra, Macromol. Symp., 102, 234 (2006).

18. S. Mohri, D. A. Rani, Y. Yamamoto, Y. Tsujita, and H. Yoshimizu, J. Polym. Sci., Part B: Polym. Phys., 42, 238 (2004).

19. K. P. O. Mahesh, M. Sivakumar, Y. Yamamoto, Y. Tsujita, H. Yoshimizu, and S. Okamoto, J. Polym. Sci., Part B: Polym. Phys., 42, 3439 (2004).

20. M. Sivakumar, K. P. O. Mahesh, Y. Yamamoto, H. Yoshimizu, and Y. Tsujita, J. Polym. Sci., Part B: Polym. Phys., 43, 1873 (2005).

21. K. P. O. Mahesh, Y. Tsujita, H. Yoshimizu, S. Okamoto, D. J. Mohan, J. Polym. Sci., Part B: Polym. Phys., 43, 2380 (2005).

22. Y. Uda, F. Kaneko, and T. Kawaguchi, Macromol. Rapid Commun., 25, 1900 (2004).

23. Y. Uda, F. Kaneko, and T. Kawaguchi, Macromolecules, 38, 3320 (2005).

24. Y. Uda, F. Kaneko, and T. Kawaguchi, Macromolecules, 38, 3380 (2005).

25. T. Nakaoki, Y. Fukuda, E. Nakajima, T. Matsuda, and T. Harada, Polym. J., 35, 430 (2003).

26. V. Vittoria, R. Russo, and F. de Candia, Polymer, 32, 3371 (1991).

27. G. Milano, V. Venditto, G. Guerra, L. Cavallo, P. Ciambelli, and D. Sannino, Chem. Mater., 13, 1506 (2001). 